QUARTERLY OF APPLIED MATHEMATICS

VOLUME LXIX, NUMBER 3

SEPTEMBER 2011, PAGES 445-464

S $0033-569 X(2011) 01216-5$

Article electronically published on April 5, 2011

\title{
ON THE CAUCHY PROBLEM FOR THE DEGASPERIS-PROCESI EQUATION
}

\author{
BY \\ GUILONG GUI (Department of Mathematics, Jiangsu University, Zhenjiang 212013, Jiangsu, \\ People's Republic of China) \\ AND \\ YUE LIU (Department of Mathematics, University of Texas, Arlington, Texas 76019)
}

Abstract. We establish here the local well-posedness for the Degasperis-Procesi equation in the Besov spaces. We also determine some blow-up criteria of the strong solutions and investigate the nonexistence of smooth solitary-wave solutions.

1. Introduction. Recently, Holm and Staley [36] studied a one-dimensional version of active fluid transport that is described by the following family of $1+1$ evolutionary equations:

$$
m_{t}+\underbrace{u m_{x}}_{\text {convection }}+\underbrace{b u_{x} m}_{\text {stretching }}=0, \text { with } u=g * m,
$$

where the fluid velocity $u(t, x)$ is defined on the real line vanishing at spatial infinity and $u=g * m$ denotes the convolution (or filtering)

$$
u(x)=\int_{\mathbb{R}} g(x-y) m(y) d y,
$$

which relates velocity $u$ to momentum density $m$ by integration against the kernel $g(x)$ over the real line. The family of equations (1.1) is characterized by the kernel $g$ and the real dimensionless constant $b$, which is the ratio of stretching to convective transport. The parameter $b$ is also the number of covariant dimensions associated with the momentum

Received November 15, 2009.

2000 Mathematics Subject Classification. Primary 35L15, 35G25, 35Q58.

Key words and phrases. Local well-posedness, blow-up, Degasperis-Procesi equation, peakons, Besov spaces.

The work of the first author was supported in part by the NSF of China under Grant 11001111, and Grants 10JDG141 and 10JDG157.

The work of the second author was partially supported by the NSF grant DMS-0906099 and the NHARP grant-003599-0001-2009. Both authors would like to thank the referee for constructive suggestions and comments.

Current address: Academy of Mathematics and Systems Science, Chinese Academy of Sciences, Beijing 100190, People's Republic of China.

E-mail address: glgui@amss.ac.cn

E-mail address: yliu@uta.edu 
density $m$. The function $g(x)$ will determine the travelling wave shape and length scale for (1.1), while the constant $b$ will provide a balance or bifurcation parameter for the nonlinear solution behavior. Here the kernel $g$ is chosen to be Green's function for the Helmholtz operator on the line; that is, $g(x)=\frac{1}{2} e^{-|x|}$. This means that $m=u-u_{x x}$. In this case, (1.1) is equivalent to the form

$$
u_{t}-u_{t x x}+(b+1) u u_{x}=b u_{x} u_{x x}+u u_{x x x}, \quad t>0, x \in \mathbb{R} .
$$

Equation (1.2) is the so-called $b$-family of equations. It was shown by Degasperis and Procesi 29] using the method of asymptotic integrability that (1.2) is completely integrable when $b=2$ or $b=3$; cf. [5, 27, 28].

If $b=2$, (1.2) becomes the Camassa-Holm $(\mathrm{CH})$ equation of the form

$$
(\mathrm{CH}) \quad u_{t}-u_{t x x}+3 u u_{x}=2 u_{x} u_{x x}+u u_{x x x}, \quad t>0, x \in \mathbb{R},
$$

modeling the unidirectional propagation of shallow water waves over a flat bottom. Again $u(t, x)$ stands for the fluid velocity at time $t$ in the spatial $x$ direction [5, 27, 38. Equation (1.3) has a bi-Hamiltonian structure [32, 51] and is completely integrable [5, 12, describing permanent and breaking waves [14, 16, 51]. It has been shown that this equation is locally well-posed [16, 50] with the initial data $u_{0} \in H^{s}(\mathbb{R})$ for $s>\frac{3}{2}$, and has global conservative solutions [2] and global dissipative solutions [3] in $H^{1}(\mathbb{R})$. On the other hand, it has global weak solutions in $H^{1}(\mathbb{R})$ 2, 54. The Camassa-Holm equation (1.3) possesses the peaked solitons [6] of the form

$$
u(x, t)=c e^{-|x-c t|}, c>0 .
$$

These peaked solitons replicate a feature [10, 15, 52] that is characteristic for waves of great height, i.e., waves of largest amplitude that are exact solutions of the governing equations for water waves. The orbital stability of the peaked solitons is proved in [23]. The explicit interaction of the peaked solitons is given in [1]. R. Danchin [24] proved the existence and uniqueness of solutions for the Camassa-Holm equation with minimal regularity assumptions on the initial data which belongs to the Besov space. Moreover, the Camassa-Holm equation has, just like the Euler equations of hydrodynamics [39] and the celebrated KdV equation [19, 40], a Riemannian geometric interpretation as geodesic flow on the diffeomorphism group [13, leading to a proof that the Least Action Principle holds 20 .

If $b=3$, (1.2) becomes the Degasperis-Procesi (DP) equation of the form

$$
u_{t}-u_{t x x}+4 u u_{x}=3 u_{x} u_{x x}+u u_{x x x}, \quad t>0, x \in \mathbb{R} .
$$

Degasperis, Holm and Hone 28, proved the formal integrability of the Degasperis-Procesi equation (1.5) by constructing a Lax pair. They also showed in [28, that (1.5) has biHamiltonian structure and an infinite sequence of conserved quantities, and that it admits exact peakon solutions which are analogous to the Camassa-Holm peakons. Peakons for either $b=2$ or $b=3$ are true solitons that interact via elastic collisions under CH dynamics, or DP dynamics, respectively. Recently Lundmark [46] showed that the Degasperis-Procesi equation (1.5) has not only peaked solitons (1.4) 28], but also shock 
peakons of the form

$$
u(t, x)=-\frac{1}{t+k} \operatorname{sgn}(x) e^{-|x|}, k>0 .
$$

Equation (1.5) can be regarded as a model for nonlinear shallow water dynamics, and its asymptotic accuracy is the same as for the Camassa-Holm shallow water equation. For the Degasperis-Procesi equation, a geometric interpretation as the geodesic flow of a right-invariant symmetric linear connection on the diffeomorphism group of the circle is available, though no metric structure exists [30].

More recently, Constantin and Lannes 21] give a rigorous proof that both the $\mathrm{CH}$ equation and the DP equation are valid approximations to the governing equations for water waves and also show the relevance of these two equations as models for the propagation of shallow water waves. Analogous to the case of the Camassa-Holm equation and the Degasperis-Procesi equation [21, one can show that the $b$-family of equations (1.2) for any $b \geq 1$ is also a model for the propagation of shallow water waves. It is worth pointing out that $b=2,3$ plays a special role in that only for these values is the corresponding equation integrable [1, 17, 21, 22, 37].

To see this rigorous justification of the derivation, one can consider the water wave equations for one-dimensional surfaces in nondimensionalized form

$$
\begin{cases}\mu \partial_{x}^{2} \psi+\partial_{z}^{2} \Psi=0, & \text { in } \Omega_{t}, \\ \partial_{z} \Psi=0, & \text { at } z=-1, \\ \partial_{t} \xi-\frac{1}{\mu}\left(-\mu \partial_{x} \xi \partial_{x} \Phi+\partial_{z} \Psi\right)=0, & \text { at } z=\epsilon \xi, \\ \partial_{t} \Psi+\frac{\epsilon}{2}\left(\partial_{x} \Psi\right)^{2}+\frac{\epsilon}{2 \mu}\left(\partial_{z} \Psi\right)^{2}=0, & \text { at } z=\epsilon \xi,\end{cases}
$$

where $x \rightarrow \epsilon \xi(t, x)$ parameterizes the elevation of the free surface at time $t, \Omega_{t}=$ $\{(x, z) ;-1<z<\epsilon \xi(t, x)\}$ is the fluid domain delimited by the free surface and the flat bottom $\{z=-1\}, \Psi(t, \cdot)$ is the velocity potential associated to the flow, and $\epsilon$ and $\mu$ are two dimensionless parameters defined by

$$
\epsilon=\frac{a}{h}, \quad \mu=\frac{h^{2}}{\lambda^{2}},
$$

where $h$ is the mean depth, $a$ is the typical amplitude, and $\lambda$ is the typical wavelength of the waves.

Consider now the so-called Camassa-Holm scaling, that is,

$$
\mu \ll 1, \quad \epsilon=O(\sqrt{\mu}) .
$$

With this scaling, one still has $\epsilon \ll 1$. The dimensionless parameter is, however, larger here than in the long wave scaling, and the nonlinear effects are therefore stronger and it is possible that a stronger nonlinearity could allow the appearance of breaking waves, which is a fundamental phenomenon in the theory of water waves that is not captured by the KdV equation.

Define the horizontal velocity $u^{\theta}(\theta \in[0,1])$ at the level line $\theta$ of the fluid domain by

$$
v \equiv u^{\theta}(x)=\left.\partial_{x} \Psi\right|_{z=(1+\epsilon \xi) \theta-1} .
$$


Let $p \in \mathbb{R}$ and $\lambda=\frac{1}{2}\left(\theta^{2}-\frac{1}{3}\right)$, with $\theta \in[0,1]$. Assume

$$
\alpha=p+\lambda, \beta=p-\frac{1}{6}+\lambda, \gamma=-\frac{2}{3} p-\frac{1}{6}-\frac{3}{2} \lambda, \delta=-\frac{9}{2} p-\frac{23}{24}-\frac{3}{2} \lambda .
$$

Under the Camassa-Holm scaling, one should have the following class of equations for $v \equiv u^{\theta}(\theta \in[0,1])$, namely

$$
v_{t}+v_{x}+\frac{3}{2} \epsilon v v_{x}+\mu\left(\alpha v_{x x x}+\beta v_{x x t}\right)=\epsilon \mu\left(\gamma v v_{x x x}+\delta v_{x} v_{x x}\right),
$$

where the $O\left(\epsilon^{4}, \eta^{2}\right)$ terms have been discarded. The vertically averaged horizontal velocity $u$ and the free surface $\xi$ satisfy

$$
\begin{aligned}
& u=u^{\theta}+\mu \lambda u_{x x}^{\theta}+2 \mu \epsilon \lambda u^{\theta} u_{x x}^{\theta}, \\
& \xi=u+\frac{\epsilon}{4} u^{2}+\mu \frac{1}{6} u_{x t}-\epsilon \mu\left(\frac{1}{6} u u_{x x}+\frac{5}{48} u_{x}^{2}\right) .
\end{aligned}
$$

By rescaling, shifting the dependent variable, and applying a Galilean transformation, the $b$-family of equations

$$
U_{t}+\kappa U_{x}+(b+1) U U_{x}-U_{t x x}=b U_{x} U_{x x}+U U_{x x x}
$$

can be obtained from (1.6) if the following conditions hold:

$$
\beta<0, \quad \alpha \neq \beta, \quad \beta=-\frac{2}{3}(b+1) \gamma, \quad \delta=b \gamma,
$$

where $p=-\frac{10-29 b}{72 b}, \lambda=-\frac{5}{18 b}-\frac{1}{9}-p$ and $\theta^{2}=\frac{11 b-10}{12 b}$.

The solution $u^{\theta}$ of (1.6) is transformed to the solution $U$ of the $b$-family of equations by

$$
U(t, x)=\frac{1}{a} u^{\theta}\left(\frac{t}{c}, \frac{x}{b_{0}}+\frac{\nu}{c} t\right),
$$

with $a=\frac{2}{3 \epsilon \kappa}(b+1)(1-\nu), b_{0}^{2}=-\frac{1}{\beta} \mu, \nu=\frac{\alpha}{\beta}$, and $c=\frac{b_{0}}{\kappa}(1-\nu)$.

Since the Degasperis-Procesi equation was derived, many papers were devoted to its study; cf. 9, 31, 34, 42, 44, 45, 46, 47, 48, 55, and the citations therein. For example, Yin proved local well-posedness to (1.5) with initial data $u_{0} \in H^{s}, s>\frac{3}{2}$ on the line [55] and the precise blow-up scenario and a blow-up result were derived. Recently, Lenells [42] classified all weak travelling wave solutions. Matsuno [48] studied multisoliton solutions and their peakon limits. Analogous to the case of the Camassa-Holm equation [11, 35], Henry [34] showed that smooth solutions to the Degasperis-Procesi equation have infinite speed of propagation (see also [49]). Coclite and Karlsen [9] also obtained global existence results for entropy weak solutions in the class of $L^{1}(\mathbb{R}) \cap B V(\mathbb{R})$ and the class of $L^{2}(\mathbb{R}) \cap$ $L^{4}(\mathbb{R})$. More generally, the authors 33 established the local well-posedness for the peakon $b$-family of equations (1.2), and demonstrated the precise blow-up scenario of strong solutions to (1.2) with certain initial data. The orbital stability of the peaked solitons of the Degasperis-Procesi equation is recently proved in 43.

Concerning the applicability of the $\mathrm{CH}$ and DP equations, Lakshmanan 41 has even argued recently that the equation might be relevant to the modeling of tsunamis (see also the discussion in A. Constantin and R. S. Johnson [18]). 
We consider here the Cauchy problem of the Degasperis-Procesi equation, that is,

$$
\text { (DP) } \begin{cases}m_{t}+u m_{x}+3 u_{x} m=0, & t>0, x \in \mathbb{R}, \\ m(0, x)=u_{0}(x)-u_{0, x x}(x), & x \in \mathbb{R},\end{cases}
$$

with $m=u-u_{x x}$. Note that if $g(x):=\frac{1}{2} e^{-|x|}, x \in \mathbb{R}$, then $\left(1-\partial_{x}^{2}\right)^{-1} f=g * f$ for all $f \in L^{2}(\mathbb{R})$ and $g * m=u$, where $*$ denotes convolution. Using this identity, and applying the pseudo-differential operator $\left(1-\partial_{x}^{2}\right)^{-1}$ to (1.7), we rewrite (1.7) as a quasi-linear nonlocal evolution equation of hyperbolic type :

$$
\begin{cases}u_{t}+u u_{x}+\partial_{x} g *\left(\frac{3}{2} u^{2}\right)=0, & t>0, x \in \mathbb{R}, \\ u(0, x)=u_{0}(x), & x \in \mathbb{R} .\end{cases}
$$

If we denote $P(D)$ as the Fourier integral operator with the Fourier multiplier $-i \xi(1+$ $\left.\xi^{2}\right)^{-1}$, then equation (1.8) becomes

$$
\begin{cases}u_{t}+u u_{x}=P(D)\left(\frac{3}{2} u^{2}\right), & t>0, x \in \mathbb{R}, \\ u(0, x)=u_{0}(x), & x \in \mathbb{R} .\end{cases}
$$

In the present paper, motivated by the method in [24, we address the question of existence and uniqueness for the initial-value problem of the Degasperis-Procesi equation (1.7) in the Besov spaces. For simplicity, we restrict ourselves to the evolution for positive times. Of course, one would get similar results for negative times: this is just a matter of changing the initial condition $u_{0}$ into $-u_{0}$.

The goal of the present paper is to study the existence of solutions for (1.7) to understand better the properties of the Degasperis-Procesi equation (1.5).

Now let us state the main result of this paper as follows, where the definition of Besov-Sobolev spaces $B_{p, r}^{s}$ and $E_{p, r}^{s}(T)$ will be given in Section 2 and Section 3.

Theorem 1.1. Suppose that $1 \leq p, r \leq+\infty$ and $s>1+\frac{1}{p}$ (or $s \geq 1+\frac{1}{p}$ if $r=1$, $1 \leq p<+\infty)$. Let $u_{0} \in B_{p, r}^{s}$. There exists a time $T>0$ such that the initial-value problem (1.8) has a unique solution $u \in E_{p, r}^{s}(T)$, and the map $u_{0} \mapsto u$ is continuous from a neighborhood of $u_{0}$ in $B_{p, r}^{s}$ into $\mathcal{C}\left([0, T] ; B_{p, r}^{s^{\prime}}\right) \cap \mathcal{C}^{1}\left([0, T] ; B_{p, r}^{s^{\prime}-1}\right)$ for every $s^{\prime}<s$ when $r=+\infty$ and $s^{\prime}=s$ whereas $r<+\infty$. If, in addition, $u_{0} \in L^{2}$, then the solution $u$ satisfies the conservation law

$$
\int_{\mathbb{R}} m(t, x) v(t, x) d x=\int_{\mathbb{R}} m_{0}(x) v_{0}(x) d x,
$$

where $m(t, x)=\left(1-\partial_{x}^{2}\right) u(t, x)$ and $v(t, x)=\left(4-\partial_{x}^{2}\right)^{-1} u(t, x)$.

REMARK 1.2. Let us recall the local existence result of $(\mathrm{CH})$ given by $\mathrm{R}$. Danchin in 24], where it is proved that if $u_{0} \in B_{p, r}^{s}$ with $s>\max \left\{1+\frac{1}{p}, \frac{3}{2}\right\}$, then there exists a time $T>0$ such that $(\mathrm{CH})$ has a unique solution $u$ in $E_{p, r}^{s}(T)$. Unlike the Camassa-Holm equation, the Degasperis-Procesi equation in (1.8) has not involved the term $\partial_{x} g \star u_{x}^{2}$, and with only the term $\partial_{x} g \star u^{2}$, which enables us only to assume the initial data $u_{0} \in B_{p, r}^{s}$ with $s>1+\frac{1}{p}$ (or $s \geq 1+\frac{1}{p}$ if $r=1,1 \leq p<+\infty$ ). 
REMark 1.3. Although the $H^{1}$-norm of the solutions in the Camassa-Holm equation is conserved, which is different from (1.10) in the Degasperis-Procesi equation, it is not necessary in the proof of Proposition 4 in [26. Hence, thanks to the (DP)'s peaked solitons of the form $u(x, t)=c e^{-|x-c t|}, c>0$ as in the Camassa-Holm equation, and following the proof of Proposition 4 in [26, one can see that (DP) is not locally wellposed in $B_{2, \infty}^{\frac{3}{2}}$ in the following sense:

There exists a global solution $u \in L^{\infty}\left(\mathbb{R}^{+} ; B_{2, \infty}^{\frac{3}{2}}\right)$ to (DP) such that for any positive $T$ and $\epsilon$ there exists a solution $v \in L^{\infty}\left(0, T ; B_{2, \infty}^{\frac{3}{2}}\right)$ with

$$
\|u(0)-v(0)\|_{B_{2, \infty}^{\frac{3}{2}}} \leq \epsilon \quad \text { and } \quad\|u-v\|_{L^{\infty}\left(0, T ; B_{2, \infty}^{\frac{3}{2}}\right)} \geq 1 .
$$

Therefore, the exponent $s=\frac{3}{2}$ is critical in the range of the Besov spaces $B_{2, r}^{s}$.

The remainder of the paper is organized as follows. Section 2 is devoted to recalling the Littlewood-Paley theory. Then the local well-posedness of the initial-value problem associated with (1.7) is established in Section 3. Some blow-up criteria of strong solutions are investigated in Section 4. Finally, nonexistence of smooth travelling-wave solutions of (1.2) is studied in Section 5.

Notation. As above and henceforth, we denote the norm of the Lebesgue space $L^{p}$ by $\|\cdot\|_{L^{p}}, 1 \leq p \leq \infty$ and the norm in the Sobolev space $H^{s}, s \in \mathbb{R}$ by $\|\cdot\|_{H^{s}}$. We denote by $*$ the spatial convolution on $\mathbb{R}$.

2. Preliminaries. For the convenience of the reader, we shall recall some basic facts on Littlewood-Paley theory and the transport equations theory; one may check [4, 7, 8 , 24, 53, for more details.

Proposition 2.1 ([7], Littlewood-Paley decomposition). Let $\mathcal{B} \stackrel{\text { def }}{=}\left\{\xi \in \mathbb{R}^{d}:|\xi| \leq \frac{4}{3}\right\}$ and $\mathcal{C} \stackrel{\text { def }}{=}\left\{\xi \in \mathbb{R}^{d}: \frac{3}{4} \leq|\xi| \leq \frac{8}{3}\right\}$. There exist two radial functions $\chi \in C_{c}^{\infty}(\mathcal{B})$ and $\varphi \in C_{c}^{\infty}(\mathcal{C})$ such that

$$
\begin{aligned}
& \chi(\xi)+\sum_{q \geq 0} \varphi\left(2^{-q} \xi\right)=1, \quad \forall \xi \in \mathbb{R}^{d}, \\
& \left|q-q^{\prime}\right| \geq 2 \Rightarrow \operatorname{Supp} \varphi\left(2^{-q} \cdot\right) \cap \operatorname{Supp} \varphi\left(2^{-q^{\prime}} \cdot\right)=\varnothing, \\
& q \geq 1 \Rightarrow \operatorname{Supp} \chi(\cdot) \cap \operatorname{Supp} \varphi\left(2^{-q} \cdot\right)=\varnothing
\end{aligned}
$$

and

$$
\frac{1}{3} \leq \chi(\xi)^{2}+\sum_{q \geq 0} \varphi\left(2^{-q} \xi\right)^{2} \leq 1, \quad \forall \xi \in \mathbb{R}^{d}
$$


Let $h \stackrel{\text { def }}{=} \mathcal{F}^{-1} \varphi$ and $\tilde{h} \stackrel{\text { def }}{=} \mathcal{F}^{-1} \chi$. Then the dyadic operators $\Delta_{q}$ and $S_{q}$ can be defined as follows:

$$
\begin{aligned}
& \Delta_{q} f \stackrel{\text { def }}{=} \varphi\left(2^{-q} D\right) f=2^{q d} \int_{\mathbb{R}^{d}} h\left(2^{q} y\right) f(x-y) d y, \quad \text { for } \quad q \geq 0, \\
& S_{q} f \stackrel{\text { def }}{=} \chi\left(2^{-q} D\right) f=\sum_{-1 \leq k \leq q-1} \Delta_{q} f=2^{q d} \int_{\mathbb{R}^{d}} \tilde{h}\left(2^{q} y\right) f(x-y) d y, \\
& \Delta_{-1} f \stackrel{\text { def }}{=} S_{0} f \quad \text { and } \quad \Delta_{q} f \stackrel{\text { def }}{=} 0 \quad \text { for } \quad q \leq-2 .
\end{aligned}
$$

Lemma 2.2 ([7], Bernstein's inequality). Let $\mathcal{B}$ be a ball with center 0 in $\mathbb{R}^{d}$ and $\mathcal{C}$ a ring with center 0 in $\mathbb{R}^{d}$. A constant $C$ exists so that, for any positive real number $\lambda$, any nonnegative integer $k$, any smooth homogeneous function $\sigma$ of degree $m$, and any couple of real numbers $(a, b)$ with $b \geq a \geq 1$, we have

$$
\begin{aligned}
& \text { Supp } \hat{u} \subset \lambda \mathcal{B} \Rightarrow \sup _{|\alpha|=k}\left\|\partial^{\alpha} u\right\|_{L^{b}} \leq C^{k+1} \lambda^{k+d\left(\frac{1}{a}-\frac{1}{b}\right)}\|u\|_{L^{a}}, \\
& \text { Supp } \hat{u} \subset \lambda \mathcal{C} \Rightarrow C^{-1-k} \lambda^{k}\|u\|_{L^{a}} \leq \sup _{|\alpha|=k}\left\|\partial^{\alpha} u\right\|_{L^{a}} \leq C^{1+k} \lambda^{k}\|u\|_{L^{a}}, \\
& \text { Supp } \hat{u} \subset \lambda \mathcal{C} \Rightarrow\|\sigma(D) u\|_{L^{b}} \leq C_{\sigma, m} \lambda^{m+d\left(\frac{1}{a}-\frac{1}{b}\right)}\|u\|_{L^{a}},
\end{aligned}
$$

for any function $u \in L^{a}$.

Definition 2.3 ([7], Besov spaces). Let $s \in \mathbb{R}, 1 \leq p, r \leq \infty$. The inhomogeneous Besov space $B_{p, r}^{s}\left(\mathbb{R}^{d}\right)\left(B_{p, r}^{s}\right.$ for short) is defined by

$$
B_{p, r}^{s}\left(\mathbb{R}^{d}\right) \stackrel{\text { def }}{=}\left\{f \in \mathcal{S}^{\prime}\left(\mathbb{R}^{d}\right):\|f\|_{B_{p, r}^{s}}<\infty\right\},
$$

where

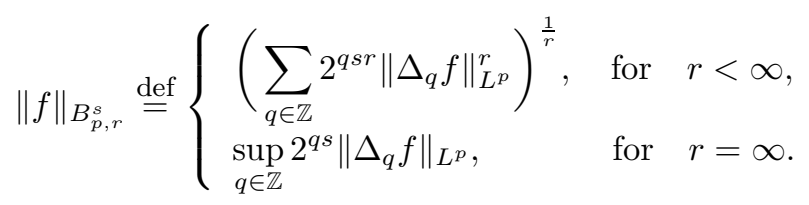

If $s=\infty, B_{p, r}^{\infty} \stackrel{\text { def }}{=} \bigcap_{s \in \mathbb{R}} B_{p, r}^{s}$.

Proposition 2.4 (24, 25]). The following properties hold.

(1) Density: if $p, r<\infty$, then $\mathcal{S}\left(\mathbb{R}^{d}\right)$ is dense in $B_{p, r}^{s}\left(\mathbb{R}^{d}\right)$.

(2) Sobolev embedding: if $p_{1} \leq p_{2}$ and $r_{1} \leq r_{2}$, then $B_{p_{1}, r_{1}}^{s} \hookrightarrow B_{p_{2}, r_{2}}^{s-d\left(\frac{1}{p_{1}}-\frac{1}{p_{2}}\right)}$. If $s_{1}<s_{2}, 1 \leq p \leq+\infty$ and $1 \leq r_{1}, r_{2} \leq+\infty$, then the embedding $B_{p, r_{2}}^{s_{2}} \hookrightarrow B_{p, r_{1}}^{s_{1}}$ is locally compact.

(3) Algebraic properties: for $s>0, B_{p, r}^{s} \cap L^{\infty}$ is an algebra. Moreover, ( $B_{p, r}^{s}$ is an algebra $) \Longleftrightarrow\left(B_{p, r}^{s} \hookrightarrow L^{\infty}\right) \Longleftrightarrow\left(s>\frac{d}{p}\right.$ or $\left(s \geq \frac{d}{p}\right.$ and $\left.\left.r=1\right)\right)$.

(4) Fatou property: if $\left(u^{n}\right)_{n \in \mathbb{N}}$ is a bounded sequence of $B_{p, r}^{s}$ which tends to $u$ in $\mathcal{S}^{\prime}$, then $u \in B_{p, r}^{s}$ and

$$
\|u\|_{B_{p, r}^{s}} \leq \lim \inf _{n \rightarrow \infty}\left\|u_{n}\right\|_{B_{p, r}^{s}} .
$$


(5) Complex interpolation: if $u \in B_{p, r}^{s} \cap B_{p, r}^{\tilde{s}}$ and $\theta \in[0,1], 1 \leq p, r \leq \infty$, then

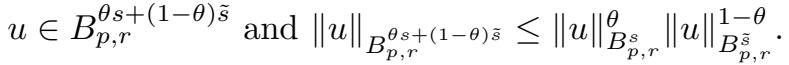

(6) Let $m \in \mathbb{R}$ and $f$ be an $S^{m}$-multiplier (that is, $f: \mathbb{R}^{d} \rightarrow \mathbb{R}$ is smooth and satisfies that for every multi-index $\alpha$, there exists a constant $C_{\alpha}$ such that $\forall \xi \in$ $\left.\mathbb{R}^{d},\left|\partial^{\alpha} f(\xi)\right| \leq C_{\alpha}(1+|\xi|)^{m-|\alpha|}\right)$. Then for all $s \in \mathbb{R}$ and $1 \leq p, r \leq \infty$, the operator $f(D)$ in continuous from $B_{p, r}^{s}$ to $B_{p, r}^{s-m}$.

Proposition 2.5 (25], Logarithmic interpolation). There is a constant $C$ such that for all $s \in \mathbb{R}, \varepsilon>0$ and $1 \leq p \leq \infty$, we have

$$
\|u\|_{B_{p, 1}^{s}} \leq C \frac{1+\varepsilon}{\varepsilon}\|u\|_{B_{p, \infty}^{s}}\left(1+\log \frac{\|u\|_{B_{p}^{s+\infty}}}{\|u\|_{B_{p, \infty}^{s}}}\right) .
$$

Lemma $2.6([24,25])$. Suppose that $(p, r) \in[1,+\infty]^{2}$ and $s>-\frac{d}{p}$. Let $v$ be a vector field such that $\nabla v$ belongs to $L^{1}\left([0, T] ; B_{p, r}^{s-1}\right)$ if $s>1+\frac{d}{p}$ or to $L^{1}\left([0, T] ; B_{p, r}^{\frac{d}{p}} \cap L^{\infty}\right)$ otherwise. Suppose also that $f_{0} \in B_{p, r}^{s}, F \in L^{1}\left([0, T] ; B_{p, r}^{s}\right)$ and that $f \in L^{\infty}\left([0, T] ; B_{p, r}^{s}\right) \cap$ $C\left([0, T] ; \mathcal{S}^{\prime}\right)$ solves the $d$-dimensional linear transport equations

$$
\text { (T) }\left\{\begin{array}{l}
\partial_{t} f+v \cdot \nabla f=F \\
\left.f\right|_{t=0}=f_{0}
\end{array}\right.
$$

Then there exists a constant $C$ depending only on $s, p$ and $d$, and such that the following statements hold:

(1) If $r=1$ or $s \neq 1+\frac{d}{p}$,

$$
\|f\|_{B_{p, r}^{s}} \leq\left\|f_{0}\right\|_{B_{p, r}^{s}}+\int_{0}^{t}\|F(\tau)\|_{B_{p, r}^{s}} d \tau+C \int_{0}^{t} V^{\prime}(\tau)\|f(\tau)\|_{B_{p, r}^{s}} d \tau,
$$

or hence,

$$
\|f\|_{B_{p, r}^{s}} \leq e^{C V(t)}\left(\left\|f_{0}\right\|_{B_{p, r}^{s}}+\int_{0}^{t} e^{-C V(\tau)}\|F(\tau)\|_{B_{p, r}^{s}} d \tau\right)
$$

with $V(t)=\int_{0}^{t}\|\nabla v(\tau)\|_{B_{p, r}^{\frac{d}{p}} \cap L^{\infty}} d \tau$ if $s<1+\frac{d}{p}$ and $V(t)=\int_{0}^{t}\|\nabla v(\tau)\|_{B_{p, r}^{s-1}} d \tau$ else.

(2) If $s \leq 1+\frac{d}{p}$ and, in addition, $\nabla f_{0} \in L^{\infty}, \nabla f \in L^{\infty}\left([0, T] \times \mathbb{R}^{d}\right)$ and $\nabla F \in$ $L^{1}\left([0, T] ; L^{\infty}\right)$, then

$$
\begin{aligned}
\|f(t)\|_{B_{p, r}^{s}} & +\|\nabla f(t)\|_{L^{\infty}} \\
\leq & e^{C V(t)}\left(\left\|f_{0}\right\|_{B_{p, r}^{s}}+\left\|\nabla f_{0}\right\|_{L^{\infty}}+\int_{0}^{t} e^{-C V(\tau)}\left(\|F(\tau)\|_{B_{p, r}^{s}}+\|\nabla F(\tau)\|_{L^{\infty}}\right) d \tau\right)
\end{aligned}
$$

with $V(t)=\int_{0}^{t}\|\nabla v(\tau)\|_{B_{p, r}^{\frac{d}{p} \cap L^{\infty}}} d \tau$.

(3) If $f=v$, then for all $s>0$, the estimate (2.1) holds with $V(t)=\int_{0}^{t}\left\|\partial_{x} u(\tau)\right\|_{L^{\infty}} d \tau$.

(4) If $r<+\infty$, then $f \in C\left([0, T] ; B_{p, r}^{s}\right)$. If $r=+\infty$, then $f \in C\left([0, T] ; B_{p, 1}^{s^{\prime}}\right)$ for all $s^{\prime}<s$. 
Lemma $2.7([25])$. Let $\left(p, p_{1}, r\right) \in[1,+\infty]^{3}$. Assume that $s>-d \min \left\{\frac{1}{p_{1}}, \frac{1}{p^{\prime}}\right\}$ with $p^{\prime}:=$ $\left(1-\frac{1}{p}\right)^{-1}$. Let $f_{0} \in B_{p, r}^{s}$ and $g \in L^{1}\left([0, T] ; B_{p, r}^{s}\right)$. Let $v$ be a time-dependent vector field such that $v \in L^{\rho}\left([0, T] ; B_{\infty, \infty}^{-M}\right)$ for some $\rho>1, M>0$ and $\nabla v \in L^{1}\left([0, T] ; B_{p_{1}, \infty}^{\frac{d}{p_{1}}} \cap L^{\infty}\right)$ if $s<1+\frac{d}{p_{1}}$, and $\nabla v \in L^{1}\left([0, T] ; B_{p_{1}, r}^{s-1}\right)$ if $s>1+\frac{d}{p_{1}}$ or $s=1+\frac{d}{p_{1}}$ and $r=1$. Then the transport equations (T) have a unique solution $f \in L^{\infty}\left([0, T] ; B_{p, r}^{s}\right) \cap\left(\bigcap_{s^{\prime}<s} \mathcal{C}\left([0, T] ; B_{p, 1}^{s^{\prime}}\right)\right)$ and the inequalities of Lemma 2.6 hold. If, moreover, $r<\infty$, then we have $f \in$ $\mathcal{C}\left([0, T] ; B_{p, r}^{s}\right)$.

Lemma 2.8 ([7, Moser-type estimates). Assuming that $1 \leq p, r \leq+\infty$, the following estimates hold:

(1) for $s>0,\|f g\|_{B_{p, r}^{s}(\mathbb{R})} \leq C\left(\|f\|_{B_{p, r}^{s}(\mathbb{R})}\|g\|_{L^{\infty}(\mathbb{R})}+\|g\|_{B_{p, r}^{s}(\mathbb{R})}\|f\|_{L^{\infty}(\mathbb{R})}\right)$;

(2) for $s_{1}, s_{2}<\frac{1}{p}$ if $r>1$ (or $s_{1}, s_{2} \leq \frac{1}{p}$ if $r=1$ ) and $s_{1}+s_{2}>0$, then

$$
\|f g\|_{B_{p, r}^{s_{1}+s_{2}-\frac{1}{p}}(\mathbb{R})} \leq C\|f\|_{B_{p, r}^{s_{1}(\mathbb{R})}}\|g\|_{B_{p, r}^{s_{2}(\mathbb{R})}},
$$

where the $C$ 's are constants independent of $f$ and $g$.

3. Local well-posedness. In this section, we shall study the local well-posedness of the Degasperis-Procesi equation in the Besov space. Uniqueness and continuity with respect to the initial data are an immediate consequence of the following result.

Proposition 3.1. Let $1 \leq p, r \leq+\infty$ and $s>1+\frac{1}{p}$ (or $s \geq 1+\frac{1}{p}$ if $r=1,1 \leq p<+\infty$ ). Let $u$ and $v$ be two given solutions of the initial-value problem (1.8) with the initial data $u_{0}, v_{0} \in B_{p, r}^{s}$ satisfying $u, v \in L^{\infty}\left([0, T] ; B_{p, r}^{s}\right) \cap C\left([0, T] ; \mathcal{S}^{\prime}\right)$. Then for every $t \in[0, T]$ :

$$
\|u(t)-v(t)\|_{B_{p, r}^{s-1}} \leq\left\|u_{0}-v_{0}\right\|_{B_{p, r}^{s-1}} e^{C \int_{0}^{t}\left(\|u(\tau)\|_{B_{p, r}^{s}}+\|v(\tau)\|_{B_{p, r}^{s}}\right) d \tau} .
$$

Proof. Denote $w \stackrel{\text { def }}{=} v-u$. It is obvious that $w \in L^{\infty}\left([0, T] ; B_{p, r}^{s}\right) \cap C\left([0, T] ; \mathcal{S}^{\prime}\right)$, which implies that $w \in C\left([0, T] ; B_{p, r}^{s-1}\right)$, and $w$ solves the following transport equation:

$$
\left\{\begin{array}{l}
\partial_{t} w+u \partial_{x} w=-w \partial_{x} v+\frac{3}{2} P(D)(w(u+v)), \\
\left.w\right|_{t=0}=w_{0} \stackrel{\text { def }}{=} v_{0}-u_{0} .
\end{array}\right.
$$

According to Lemma 2.6], we have

$$
\begin{aligned}
& e^{-C \int_{0}^{t}\left\|\partial_{x} u(\tau)\right\|_{B_{p}^{s-r}}^{s-1} d \tau}\|w(t)\|_{B_{p}^{s-1}} \\
& \leq\left\|w_{0}\right\|_{B_{p, r}^{s-1}}+C \int_{0}^{t} e^{-C \int_{0}^{\tau}\left\|\partial_{x} u\left(\tau^{\prime}\right)\right\|_{B_{p, r}^{s-1}} d \tau^{\prime}} \\
& \quad \times\left(\left\|\left(w \partial_{x} v\right)(\tau)\right\|_{B_{p, r}^{s-1}}+\|P(D)(w(u+v))(\tau)\|_{B_{p, r}^{s-1}}\right) d \tau .
\end{aligned}
$$

For $s>1+\frac{1}{p}$ (or $s \geq 1+\frac{1}{p}$ if $r=1,1 \leq p<+\infty$ ), $B_{p, r}^{s-1} \subset L^{\infty}$ is an algebra according to Proposition 2.4. so we have

$$
\left\|w \partial_{x} v\right\|_{B_{p, r}^{s-1}} \leq C\|w\|_{B_{p, r}^{s-1}}\left\|\partial_{x} v\right\|_{B_{p, r}^{s-1}} \leq C\|w\|_{B_{p, r}^{s-1}}\|v\|_{B_{p, r}^{s}} .
$$


On the other hand, thanks to $P(\xi)$ being an $S^{-1}$-multiple, applying Proposition 2.4 yields

$$
\|P(D)(w(u+v))\|_{B_{p, r}^{s-1}} \leq C\|w(u+v)\|_{B_{p, r}^{s-2}} \leq C\|w\|_{B_{p, r}^{s-1}}\left(\|u\|_{B_{p, r}^{s-1}}+\|v\|_{B_{p, r}^{s-1}}\right),
$$

which, together with (3.3) and (3.2), gives

$$
\begin{aligned}
& e^{-C \int_{0}^{t}\left\|\partial_{x} u(\tau)\right\|_{B_{p, r}^{s-1}} d \tau}\|w(t)\|_{B_{p, r}^{s-1}} \\
& \quad \leq\left\|w_{0}\right\|_{B_{p, r}^{s-1}}+C \int_{0}^{t} e^{-C \int_{0}^{\tau}\left\|\partial_{x} u\left(\tau^{\prime}\right)\right\|_{B_{p, r}^{s-1}} d \tau^{\prime}}\|w(\tau)\|_{B_{p, r}^{s-1}}\left(\|u(\tau)\|_{B_{p, r}^{s-1}}+\|v(\tau)\|_{B_{p, r}^{s, r}}\right) d \tau .
\end{aligned}
$$

Hence, applying the Gronwall inequality, we reach (3.1).

Definition 3.2. For $T>0, s \in \mathbb{R}$ and $1 \leq p \leq+\infty$, we set

$$
\begin{aligned}
& E_{p, r}^{s}(T) \stackrel{\text { def }}{=} \mathcal{C}\left([0, T] ; B_{p, r}^{s}\right) \cap \mathcal{C}^{1}\left([0, T] ; B_{p, r}^{s-1}\right) \quad \text { if } \quad r<+\infty \\
& E_{p, \infty}^{s}(T) \stackrel{\text { def }}{=} L^{\infty}\left([0, T] ; B_{p, \infty}^{s}\right) \cap \operatorname{Lip}\left([0, T] ; B_{p, \infty}^{s-1}\right)
\end{aligned}
$$

and $E_{p, r}^{s} \stackrel{\text { def }}{=} \bigcap_{T>0} E_{p, r}^{s}(T)$.

Now let us start the proof of Theorem 1.1 which is motivated by the proof of a local existence theorem about $(\mathrm{CH})$ in [24. Firstly, we shall use the classical Friedrichs regularization method to construct the approximate solutions to (DP).

LEMma 3.3. Let $p, r$ and $s$ be as in the statement of Theorem[1.1. Assume that $u^{(0)}:=0$. There exists a sequence of smooth functions $\left(u^{(n)}\right)_{n \in \mathbb{N}} \in \mathcal{C}\left(\mathbb{R}^{+} ; B_{p, r}^{\infty}\right)$ solving the following linear transport equation by induction:

$$
\left(T_{n}\right) \begin{cases}\left(\partial_{t}+u^{(n)} \partial_{x}\right) u^{(n+1)}=P(D)\left(\frac{3}{2}\left(u^{(n)}\right)^{2}\right), & t>0, x \in \mathbb{R}, \\ \left.u^{(n+1)}\right|_{t=0}=u_{0}^{(n+1)}(x)=S_{n+1} u_{0}, & x \in \mathbb{R} .\end{cases}
$$

Moreover, there is a positive $T$ such that the solutions satisfy the following properties:

(1) $\left(u^{(n)}\right)_{n \in \mathbb{N}}$ is uniformly bounded in $E_{p, r}^{s}(T)$.

(2) $\left(u^{(n)}\right)_{n \in \mathbb{N}}$ is a Cauchy sequence in $\mathcal{C}\left([0, T] ; B_{p, r}^{s-1}\right)$.

Proof. Since all the data $S_{n+1} u_{0}$ belong to $B_{p, r}^{\infty}$, Lemma 2.7 enables us to show by induction that for all $n \in \mathbb{N}$, the equation $\left(T_{n}\right)$ has a global solution which belongs to $\mathcal{C}\left(\mathbb{R} ; B_{p, r}^{\infty}\right)$. Thanks to Lemma 2.6 and the proof of Proposition 3.1, we have the following inequality for all $n \in \mathbb{N}$ :

$$
\begin{aligned}
& e^{-C \int_{0}^{t}\left\|u^{(n)}\left(\tau^{\prime}\right)\right\|_{B_{p, r}^{s}} d \tau^{\prime}}\left\|u^{(n+1)}(t)\right\|_{B_{p, r}^{s}} \\
& \quad \leq\left\|u_{0}\right\|_{B_{p, r}^{s}}+C \int_{0}^{t} e^{-C \int_{0}^{\tau}\left\|u^{(n)}\left(\tau^{\prime}\right)\right\|_{B_{p, r}^{s}} d \tau^{\prime}}\left\|u^{(n)}(\tau)\right\|_{B_{p, r}^{s}}^{2} d \tau .
\end{aligned}
$$

Let us choose a $T>0$ such that $2 C\left\|u_{0}\right\|_{B_{p, r}^{s}} T<1$ and suppose by induction that

$$
\left\|u^{(n)}(t)\right\|_{B_{p, r}^{s}} \leq \frac{\left\|u_{0}\right\|_{B_{p, r}^{s}}}{1-2 C\left\|u_{0}\right\|_{B_{p, r}^{s}} t} \quad \forall t \in[0, T] .
$$


Inserting (3.7) into (3.6) yields

$$
\begin{aligned}
& \left\|u^{(n+1)}(t)\right\|_{B_{p, r}^{s}} \\
& \quad \leq \frac{1}{\sqrt{1-2 C\left\|u_{0}\right\|_{B_{p, r}^{s} t} t}}\left(\left\|u_{0}\right\|_{B_{p, r}^{s}}+C\left\|u_{0}\right\|_{B_{p, r}^{s}}^{2} \int_{0}^{t} \frac{d \tau}{\left(1-2 C\left\|u_{0}\right\|_{B_{p, r}^{s}} \tau\right)^{\frac{3}{2}}}\right) \\
& \quad \leq \frac{\left\|u_{0}\right\|_{B_{p, r}^{s}}}{1-2 C\left\|u_{0}\right\|_{B_{p, r}^{s} t} t} .
\end{aligned}
$$

Therefore, $\left(u^{(n)}\right)_{n \in \mathbb{N}}$ is uniformly bounded in $\mathcal{C}\left([0, T] ; B_{p, r}^{s}\right)$. Since $B_{p, r}^{s-1}$ with $s>1+\frac{1}{p}$ (or $s \geq 1+\frac{1}{p}$ if $r=1,1 \leq p<+\infty$ ) is an algebra, one can see that $u^{(n)} \partial_{x} u^{(n+1)}$ and $P(D)\left(\frac{3}{2}\left(u^{(n)}\right)^{2}\right)$ are uniformly bounded in $\mathcal{C}\left([0, T] ; B_{p, r}^{s-1}\right)$. Hence, $\partial_{t} u^{(n+1)}(:=$ $\left.-u^{(n)} \partial_{x} u^{(n+1)}+P(D)\left(\frac{3}{2}\left(u^{(n)}\right)^{2}\right)\right)$ belongs to $\mathcal{C}\left([0, T] ; B_{p, r}^{s-1}\right)$, which yields that the sequence $\left(u^{(n)}\right)_{n \in \mathbb{N}}$ is uniformly bounded in $E_{p, r}^{s}(T)$.

Next we are going to show that $\left(u^{(n)}\right)_{n \in \mathbb{N}}$ is a Cauchy sequence in $\mathcal{C}\left([0, T] ; B_{p, r}^{s-1}\right)$. According to (3.5), we deduce that, for all $m, n \in \mathbb{N}$,

$$
\begin{aligned}
\left(\partial_{t}+\right. & \left.u^{(n+m)} \partial_{x}\right)\left(u^{(n+m+1)}-u^{(n+1)}\right) \\
& =\left(u^{(n)}-u^{(n+m)}\right) \partial_{x} u^{(n+1)}+\frac{3}{2} P(D)\left(\left(u^{(n+m)}-u^{(n)}\right)\left(u^{(n+m)}+u^{(n)}\right)\right) .
\end{aligned}
$$

Lemma 2.6 applied again, together with the fact that $B_{p, r}^{s-1}$ is an algebra and the property of the operator $P(D)$ as given in (3.4), yields for every $t \in[0, T]$,

$$
\begin{aligned}
& \left\|\left(u^{(n+m+1)}-u^{(n+1)}\right)(t)\right\|_{B_{p, r}^{s-1}} \\
& \leq\left\|u_{0}^{(n+m+1)}-u_{0}^{(n+1)}\right\|_{B_{p, r}^{s-1}} e^{C \int_{0}^{t}\left\|u^{(n+m)}\left(\tau^{\prime}\right)\right\|_{B_{p, r}^{s}, r} d \tau^{\prime}} \\
& \quad+C \int_{0}^{t} e^{C \int_{\tau}^{t}\left\|u^{(n+m)}\left(\tau^{\prime}\right)\right\|_{B_{p}^{s}, r}^{s} d \tau^{\prime}}\left\|\left(u^{(n+m)}-u^{(n)}\right)(\tau)\right\|_{B_{p, r}^{s-1}} \\
& \quad \times\left(\left\|u^{(n)}(\tau)\right\|_{B_{p, r}^{s}}+\left\|u^{(n+1)}(\tau)\right\|_{B_{p, r}^{s}}+\left\|u^{(n+m)}(\tau)\right\|_{B_{p, r}^{s}}\right) d \tau .
\end{aligned}
$$

Since $\left(u^{(n)}\right)_{n \in \mathbb{N}}$ is uniformly bounded in $E_{p, r}^{s}(T)$ and

$$
u_{0}^{n+m+1}-u_{0}^{n+1}=S_{n+m+1} u_{0}-S_{n+1} u_{0}=\sum_{q=n+1}^{n+m} \Delta_{q} u_{0},
$$

we get a constant $C_{T}$ independent of $n, m$ such that for all $t \in[0, T]$,

$$
\left\|\left(u^{(n+m+1)}-u^{(n+1)}\right)(t)\right\|_{B_{p, r}^{s-1}} \leq C_{T}\left(2^{-n}+\int_{0}^{t}\left\|\left(u^{(n+m)}-u^{(n)}\right)(\tau)\right\|_{B_{p, r}^{s-1}} d \tau\right) .
$$

Arguing by induction, one can easily prove that

$$
\left\|u^{(n+m+1)}-u^{(n)}\right\|_{L_{T}^{\infty}\left(B_{p, r}^{s-1}\right)} \leq \frac{\left(T C_{T}\right)^{n+1}}{(n+1) !}\left\|u^{(m)}\right\|_{L_{T}^{\infty}\left(B_{p, r}^{s}\right)}+C_{T} \sum_{k=0}^{n} 2^{-(n-k)} \frac{\left(T C_{T}\right)^{k}}{k !} .
$$

As $\left\|u^{(m)}\right\|_{L_{T}^{\infty}\left(B_{p, r}^{s}\right)}$ can be bounded independently of $m$, we conclude the existence of some new constant $C_{T}^{\prime}$ independent of $n, m$ such that

$$
\left\|u^{(n+m+1)}-u^{(n+1)}\right\|_{L_{T}^{\infty}\left(B_{p, r}^{s-1}\right)} \leq C_{T}^{\prime} 2^{-n} .
$$


Hence $\left(u^{(n)}\right)_{n \in \mathbb{N}}$ is a Cauchy sequence in $\mathcal{C}\left([0, T] ; B_{p, r}^{s-1}\right)$.

Proof of Theorem 1.1. Thanks to Lemma 3.3, we get that $\left(u^{(n)}\right)_{n \in \mathbb{N}}$ is a Cauchy sequence in $\mathcal{C}\left([0, T] ; B_{p, r}^{s-1}\right)$, so, it converges to some limit function $u \in \mathcal{C}\left([0, T] ; B_{p, r}^{s-1}\right)$. We now have to check that $u$ belongs to $E_{p, r}^{s}(T)$ and solves the (DP) equation. Since $\left(u^{(n)}\right)_{n \in \mathbb{N}}$ is uniformly bounded in $L^{\infty}\left([0, T] ; B_{p, r}^{s}\right)$ according to Lemma 3.3 , the Fatou property for Besov spaces (Proposition 2.4) guarantees that $u$ also belongs to $L^{\infty}\left([0, T] ; B_{p, r}^{s}\right)$.

On the other hand, as $\left(u^{(n)}\right)_{n \in \mathbb{N}}$ converges to $u$ in $\mathcal{C}\left([0, T] ; B_{p, r}^{s-1}\right)$, an interpolation argument insures that the convergence holds in $\mathcal{C}\left([0, T] ; B_{p, r}^{s^{\prime}}\right)$, for any $s^{\prime}<s$. It is then easy to pass to the limit in the equation $\left(T_{n}\right)$ and to conclude that $u$ is indeed a solution to (DP). Now, because $u$ belongs to $L^{\infty}\left([0, T] ; B_{p, r}^{s}\right)$, the right-hand side of the equation

$$
\partial_{t} u+u \partial_{x} u=P(D)\left(\frac{3}{2} u^{2}\right)
$$

also belongs to $L^{\infty}\left([0, T] ; B_{p, r}^{s}\right)$. In particular, for the case $r<\infty$, Lemma 2.7 enables us to conclude that $u \in \mathcal{C}\left([0, T] ; B_{p, r}^{s^{\prime}}\right)$. Finally, using the equation again, we see that $\partial_{t} u \in \mathcal{C}\left([0, T] ; B_{p, r}^{s-1}\right)$ if $r<\infty$, and in $L^{\infty}\left([0, T] ; B_{p, r}^{s-1}\right)$ otherwise. Therefore, $u$ belongs to $E_{p, r}^{s}(T)$.

For the continuity in $E_{p, r}^{s}(T)$, if $v_{0}$ is in a small neighborhood of $u_{0}$ in $B_{p, r}^{s}$ with $s>1+\frac{1}{p}$, the existence of a solution $v \in E_{p, r}^{s}(T)$ to (DP) with initial data $v_{0}$ can be proved by using the arguments above. Proposition 3.1, together with the interpolation inequalities, ensures the continuity with respect to the initial data in $E_{p, r}^{s^{\prime}}(T)$ (with $\left.s^{\prime}<s\right)$ and then in $E_{p, r}^{s}(T)$. In fact, a standard use of a sequence of viscosity approximate solutions $\left(u_{\epsilon}\right)_{\epsilon>0}$ for $(\mathrm{DP})$ which converges uniformly in $\mathcal{C}\left([0, T] ; B_{p, r}^{s}\right) \cap \mathcal{C}^{1}\left([0, T] ; B_{p, r}^{s-1}\right)$ gives that $u$ also belongs to $\mathcal{C}\left([0, T] ; B_{p, r}^{s}\right) \cap \mathcal{C}^{1}\left([0, T] ; B_{p, r}^{s-1}\right)$. For the continuity in the critical space $E_{p, 1}^{1+\frac{1}{p}}(T)$, one may check the proof of Proposition 2 in [26] (where $p=2$ ), which, together with Proposition 3.1 and Proposition 2.5. yields the complete proof of the continuity.

Let us now prove (1.10). Applying a simple density argument, we only need to show that the conservation law (1.10) holds with some $s \geq 3$. Here we assume $s=3$ to complete the proof of the above theorem. By (1.7), and applying the Plancherel identity, we have

$$
\begin{aligned}
\frac{1}{2} \frac{d}{d t} \int_{\mathbb{R}} m v d x & =\frac{1}{2} \int_{\mathbb{R}} m_{t} v d x+\frac{1}{2} \int_{\mathbb{R}} m v_{t} d x=\int_{\mathbb{R}} m_{t} v d x \\
& =-\int_{\mathbb{R}} v m_{x} u d x-3 \int_{\mathbb{R}} v m u_{x} d x .
\end{aligned}
$$

According to the proof of Lemma 3.1 in [4], we claim that

$$
\int_{\mathbb{R}} v m_{x} u+3 \int_{\mathbb{R}} v m u_{x} d x=0 .
$$


In fact, using the relations $m=u-u_{x x}$ and $u=4 v-v_{x x}$, this yields that

$$
\begin{aligned}
\int_{\mathbb{R}} v(m u)_{x} d x & =-\int_{\mathbb{R}} v_{x} m u d x=-\int_{\mathbb{R}} v_{x} u^{2} d x-\int_{\mathbb{R}}\left(v_{x} u\right) u_{x} d x \\
& =-\int_{\mathbb{R}} v_{x} u^{2} d x+\frac{1}{2} \int_{\mathbb{R}} v_{x x x} u^{2} d x-\int_{\mathbb{R}} v_{x} \\
& =-\int_{\mathbb{R}} v_{x} u^{2} d x+\frac{1}{2} \int_{\mathbb{R}}\left(4 v_{x}-u_{x}\right) u^{2} d x-\int_{\mathbb{R}} v_{x} u_{x}^{2} d x \\
& =\int_{\mathbb{R}} v_{x} u^{2} d x-\int_{\mathbb{R}} v_{x} u_{x}^{2} d x
\end{aligned}
$$

and

Therefore,

$$
2 \int_{\mathbb{R}} v m u_{x} d x=-\int_{\mathbb{R}} v_{x} u^{2} d x+\int_{\mathbb{R}} v_{x} u_{x}^{2} d x
$$

$$
\int_{\mathbb{R}} v m_{x} u+3 \int_{\mathbb{R}} v m u_{x} d x=\int_{\mathbb{R}} v(m u)_{x} d x+2 \int_{\mathbb{R}} v m u_{x} d x=0,
$$

which, together with (3.8), implies (1.10).

4. Blow-up time. Firstly, we define the lifespan of the solution of (DP) as follows:

Definition 4.1. Let $u_{0} \in B_{p, r}^{s}$. We define the lifespan $T_{u_{0}}^{\star}$ of the solution of (DP) with the initial data $u_{0}$ as the supremum of positive times $T$ such that (DP) has a solution $u \in E_{p, r}^{s}(T)$ on $[0, T] \times \mathbb{R}$.

Now let us state the first blow-up criterion theorem:

TheOREm 4.2. Let $u_{0}$ be as in Theorem 1.1 and $u$ be the corresponding solution to (DP). Then

$$
T_{u_{0}}^{\star}<\infty \Rightarrow \int_{0}^{T_{u_{0}}^{\star}}\left\|\partial_{x} u(\tau)\right\|_{L^{\infty}} d \tau=\infty
$$

To prove Theorem 4.2, we need to estimate the norms $\|u(t)\|_{B_{p, r}^{s}}$ and $\|u(t)\|_{\text {Lip }}$.

Lemma 4.3. Assume that $1 \leq p, r \leq+\infty$ and $s>1$. Let $u \in L^{\infty}\left([0, T] ; B_{p, r}^{s} \cap \operatorname{Lip}\right)$ solving (DP) with the initial data $u_{0} \in B_{p, r}^{s} \cap$ Lip. There exists a constant $C$ depending only on $s$ and $p$ and a universal constant $C_{1}$ such that for all $t \in[0, T)$, we have

$$
\|u(t)\|_{B_{p, r}^{s}} \leq\left\|u_{0}\right\|_{B_{p, r}^{s}} e^{C \int_{0}^{t}\|u(\tau)\|_{\text {Lip }} d \tau}
$$

and

$$
\|u(t)\|_{L i p} \leq\left\|u_{0}\right\|_{\text {Lip }} e^{C_{1} \int_{0}^{t}\left\|\partial_{x} u(\tau)\right\|_{L^{\infty} d \tau}} .
$$

Proof. Since $u$ solves the following transport equation,

$$
\partial_{t} u+u \partial_{x} u=P(D)\left(\frac{3}{2} u^{2}\right),
$$

we have

$$
\begin{aligned}
\|u\|_{B_{p, r}^{s}} \leq & e^{C \int_{0}^{t}\left\|\partial_{x} u(\tau)\right\|_{L} \infty d \tau} \\
& \times\left(\left\|u_{0}\right\|_{B_{p, r}^{s}}+C \int_{0}^{t} e^{-C \int_{0}^{\tau}\left\|\partial_{x} u\left(\tau^{\prime}\right)\right\|_{L} \infty d \tau^{\prime}}\left\|P(D)\left(u^{2}\right)(\tau)\right\|_{B_{p, r}^{s}} d \tau\right),
\end{aligned}
$$


which follows from Lemma 2.6 Moreover, the Moser-type estimate gives

$$
\left\|P(D) u^{2}\right\|_{B_{p, r}^{s}} \leq C\left\|u^{2}\right\|_{B_{p, r}^{s-1}} \leq C\|u\|_{B_{p, r}^{s-1}}\|u\|_{L^{\infty}} \leq C\|u\|_{B_{p, r}^{s}}\|u\|_{L^{\infty}}
$$

Hence,

$$
e^{-C \int_{0}^{t}\left\|\partial_{x} u(\tau)\right\|_{L^{\infty}} d \tau}\|u\|_{B_{p, r}^{s}} \leq\left\|u_{0}\right\|_{B_{p, r}^{s}}+C \int_{0}^{t} e^{-C \int_{0}^{\tau}\left\|\partial_{x} u\left(\tau^{\prime}\right)\right\|_{L^{\infty} d \tau^{\prime}}}\|u\|_{B_{p, r}^{s}}\|u\|_{L^{\infty}} d \tau,
$$

which completes the proof of (4.1) according to the Gronwall inequality.

On the other hand, it is easy to verify the following $L^{\infty}$ estimate for transport equations:

$$
\begin{aligned}
\|u(t)\|_{L^{\infty}} & \leq\left\|u_{0}\right\|_{L^{\infty}}+C_{1} \int_{0}^{t}\left\|P(D)\left(u^{2}\right)(\tau)\right\|_{L^{\infty}} d \tau \\
& \leq\left\|u_{0}\right\|_{L^{\infty}}+C_{1} \int_{0}^{t}\|u(\tau)\|_{L^{\infty}}\left\|\partial_{x} u(\tau)\right\|_{L^{\infty}} d \tau .
\end{aligned}
$$

By differentiating once the (DP) equation with respect to $x$, we have

$$
\partial_{t}\left(\partial_{x} u\right)+u \partial_{x}\left(\partial_{x} u\right)=-\left(\partial_{x} u\right)^{2}+\partial_{x} P(D)\left(\frac{3}{2} u^{2}\right) .
$$

Applying the $L^{\infty}$ estimate for transport equations again, we prove that

$$
\begin{aligned}
\left\|\partial_{x} u(t)\right\|_{L^{\infty}} & \leq\left\|\partial_{x} u_{0}\right\|_{L^{\infty}}+C_{1} \int_{0}^{t}\left\|\left(\left(\partial_{x} u\right)^{2}+P(D)\left(u \partial_{x} u\right)\right)(\tau)\right\|_{L^{\infty}} d \tau \\
& \leq\left\|\partial_{x} u_{0}\right\|_{L^{\infty}}+C_{1} \int_{0}^{t}\left(\|u(\tau)\|_{L^{\infty}}+\left\|\partial_{x} u(\tau)\right\|_{L^{\infty}}\right)\left\|\partial_{x} u(\tau)\right\|_{L^{\infty}} d \tau \\
& \leq\left\|\partial_{x} u_{0}\right\|_{L^{\infty}}+C_{1} \int_{0}^{t}\|u(\tau)\|_{L i p}\left\|\partial_{x} u(\tau)\right\|_{L^{\infty}} d \tau
\end{aligned}
$$

which, together with (4.3), implies (4.2).

Proof of Theorem 4.2, Let $u \in \bigcap_{0<T<T_{u_{0}}^{\star}} E_{p, r}^{s}(T)$ be such that

$$
\int_{0}^{T_{u_{0}}^{\star}}\left\|\partial_{x} u\right\|_{L^{\infty}} d \tau<+\infty
$$

According to the inequality (4.2), $\int_{0}^{T_{u_{0}}^{\star}}\|u\|_{\text {Lip }} d \tau<+\infty$. Hence, (4.1) yields that

$$
\|u(t)\|_{B_{p, r}^{s}} \leq M_{T_{u_{0}}^{\star}} \stackrel{\text { def }}{=}\left\|u_{0}\right\|_{B_{p, r}^{s}} e^{C \int_{0}^{T_{u_{0}}^{\star}}\|u(\tau)\|_{\text {Lip }} d \tau}<+\infty
$$

for all $t \in\left[0, T_{u_{0}}^{\star}\right)$. Let $\epsilon>0$ be sufficiently small such that $2 C^{2} \epsilon M_{T_{u_{0}}^{\star}}<1$, where $C$ is the constant used in the proof of Theorem 1.1. Therefore, we have a solution $\tilde{u}(t) \in E_{p, r}^{s}(\epsilon)$ to (DP) with the initial data $u\left(T_{u_{0}}^{\star}-\frac{\epsilon}{2}\right)$. By the uniqueness, $\tilde{u}(t)=u\left(t+T_{u_{0}}^{\star}-\frac{\epsilon}{2}\right)$ on $\left[0, \frac{\epsilon}{2}\right)$ so that $\tilde{u}$ extends the solution $u$ beyond $T_{u_{0}}^{\star}$, which completes the proof of Theorem 4.2 .

More precisely, thanks to the Logarithmic interpolation (Proposition 2.5), we have

TheOREM 4.4. Under the assumption that $u_{0} \in B_{p, r}^{s} \cap L^{2}$ with $1 \leq p, r \leq+\infty$ and $s>1+\frac{1}{p}$ (or $s \geq 1+\frac{1}{p}$ if $r=1,1 \leq p<+\infty$ ), we have

$$
T_{u_{0}}^{\star}<\infty \Rightarrow \int_{0}^{T_{u_{0}}^{\star}}\left\|\partial_{x} u(\tau)\right\|_{B_{\infty, \infty}^{0}} d \tau=\infty
$$


Proof. Thanks to Proposition 2.4 and Proposition 2.5] we have for $s>1+\frac{1}{p}$ (or $s \geq 1+\frac{1}{p}$ if $\left.r=1,1 \leq p<+\infty\right)$

$$
\begin{aligned}
\left\|\partial_{x} u\right\|_{L^{\infty}} & \leq C\left\|\partial_{x} u\right\|_{B_{\infty, 1}^{0}} \leq C \frac{s-\frac{1}{p}}{s-1-\frac{1}{p}}\left\|\partial_{x} u\right\|_{B_{\infty, \infty}^{0}}\left(1+\log \frac{\left\|\partial_{x} u\right\|_{B_{\infty, \infty}^{s-1-\frac{1}{p}}}}{\left\|\partial_{x} u\right\|_{B_{\infty, \infty}^{0}}^{0}}\right) \\
& \leq C(s, p)\left(1+\left\|\partial_{x} u\right\|_{B_{\infty, \infty}^{0}} \log \left(e+\left\|\partial_{x} u\right\|_{B_{p, r}^{s-1}}\right)\right) .
\end{aligned}
$$

Hence,

$$
\|u\|_{\text {Lip }}=\|u\|_{L^{\infty}}+\left\|\partial_{x} u\right\|_{L^{\infty}} \leq C\left(1+\|u\|_{B_{\infty, \infty}^{1}} \log \left(e+\|u\|_{B_{p, r}^{s}}\right)\right) .
$$

Plugging this into (4.1), we get

$$
\|u(t)\|_{B_{p, r}^{s}} \leq\left\|u_{0}\right\|_{B_{p, r}^{s}} e^{C t+C \int_{0}^{t}\|u\|_{B_{\infty}^{1}, \infty}^{1} \log \left(e+\|u\|_{B_{p, r}^{s}}^{s}\right) d \tau} .
$$

Therefore,

$$
\log \left(e+\|u(t)\|_{B_{p, r}^{s}}\right) \leq \log \left(e+\left\|u_{0}\right\|_{B_{p, r}^{s}}\right)+C t+C \int_{0}^{t}\|u\|_{B_{\infty, \infty}^{1}} \log \left(e+\|u\|_{B_{p, r}^{s}}\right) d \tau .
$$

The Gronwall inequality yields

$$
\log \left(e+\|u(t)\|_{B_{p, r}^{s}}\right) \leq\left(\log \left(e+\left\|u_{0}\right\|_{B_{p, r}^{s}}\right)+C t\right) e^{\int_{0}^{t}\|u\|_{B_{\infty}^{1}, \infty} d \tau} .
$$

If $\int_{0}^{T}\left\|\partial_{x} u(\tau)\right\|_{B_{\infty, \infty}^{0}} d \tau<\infty$, it follows from (4.2) that

$$
\int_{0}^{T}\|u\|_{B_{\infty, \infty}^{1}} d \tau \leq C \int_{0}^{T}\|u\|_{L i p} d \tau<\infty
$$

which immediately follows by $u \in L^{\infty}\left([0, T] ; B_{p, r}^{s}\right)$. Arguing as in the proof of Theorem 4.2 completes the proof of Theorem 4.4

Similar to the Camassa-Holm equation [24, for the Degasperis-Procesi equation, we can get the lower semicontinuity of existence time with respect to sufficiently smooth initial data.

Theorem 4.5. Assume that $1 \leq p, r \leq+\infty, s>1+\frac{1}{p}$ (or $s \geq 1+\frac{1}{p}$ if $r=1$, $1 \leq p<+\infty)$ and $v_{0} \in B_{p, r}^{s}$. Let $u_{0} \in B_{p, 1}^{2+\frac{1}{p}}$ generate the solution $u(t, x)$ to (DP) with the lifespan $T_{u_{0}}^{\star}$ and $T<T_{u_{0}}^{\star}$. Then there exists a constant $C=C(p)$ such that if

$$
\left\|u_{0}-v_{0}\right\|_{B_{p, 1}^{1+\frac{1}{p}}}<\frac{1}{C \int_{0}^{T} \exp \left\{C \int_{0}^{\tau}\left\|u\left(\tau^{\prime}\right)\right\|_{B_{p, 1}^{2+\frac{1}{p}}} d \tau^{\prime}\right\} d \tau}
$$

then (DP) has a unique solution $v \in E_{p, r}^{s}(T)$ with the initial data $v_{0}$.

Proof. Thanks to Theorem 1.1, $v_{0}$ generates a unique maximal solution $v$ to the (DP) equation with the lifespan $T_{v_{0}}^{\star}>0$. Denoting $w \stackrel{\text { def }}{=} v-u$ once again, we get that $w$ solves the following transport equation:

$$
\left\{\begin{array}{l}
\partial_{t} w+(u+w) \partial_{x} w=-w \partial_{x} u+\frac{3}{2} P(D)\left(w^{2}+2 w u\right), \\
\left.w\right|_{t=0}=w_{0} \stackrel{\text { def }}{=} v_{0}-u_{0} .
\end{array}\right.
$$


Denote $T^{\star} \stackrel{\text { def }}{=} \min \left\{T_{u_{0}}^{\star}, T_{v_{0}}^{\star}\right\}$. Hence, an application of Lemma 2.6 implies that for every $0 \leq t<T^{\star}$,

$$
\begin{aligned}
\|w\|_{B_{p, 1}^{1+\frac{1}{p}}} \leq & \left\|w_{0}\right\|_{B_{p, 1}^{1+\frac{1}{p}}}+\int_{0}^{t}\left\|w \partial_{x} u\right\|_{B_{p, 1}^{1+\frac{1}{p}}} d \tau+\int_{0}^{t} \frac{3}{2}\left\|P(D)\left(w^{2}+2 w u\right)\right\|_{B_{p, 1}^{1+\frac{1}{p}}} d \tau \\
& +C \int_{0}^{t}\left(\left\|\partial_{x} u\right\|_{B_{p, 1}^{\frac{1}{p}}}+\left\|\partial_{x} w\right\|_{B_{p, 1}^{\frac{1}{p}}}\right)\|w\|_{B_{p, 1}^{1+\frac{1}{p}}} d \tau .
\end{aligned}
$$

Thanks to Lemma 2.8 and the fact that $L^{\infty} \hookrightarrow B_{p, 1}^{\frac{1}{p}}$, we have

$$
\|w\|_{B_{p, 1}^{1+\frac{1}{p}}} \leq\left\|w_{0}\right\|_{B_{p, 1}^{1+\frac{1}{p}}}+C \int_{0}^{t}\left(\left\|\partial_{x} u\right\|_{B_{p, 1}^{1+\frac{1}{p}}}+\|w\|_{B_{p, 1}^{1+\frac{1}{p}}}\right)\|w\|_{B_{p, 1}^{1+\frac{1}{p}}} d \tau .
$$

According to Gronwall's inequality, we have

$$
\|w\|_{B_{p, 1}^{1+\frac{1}{p}}} \leq\left\|w_{0}\right\|_{B_{p, 1}^{1+\frac{1}{p}}} \exp \left(C \int_{0}^{t}\left(\|u\|_{B_{p, 1}^{2+\frac{1}{p}}}+\|w\|_{B_{p, 1}^{1+\frac{1}{p}}}\right) d \tau\right) .
$$

Let

$$
T^{\star \star} \stackrel{\text { def }}{=} \min \left\{t: 0 \leq t \leq T_{u_{0}}^{\star}, C\left\|w_{0}\right\|_{B_{p, 1}^{1+\frac{1}{p}}} \int_{0}^{t} \exp \left(C \int_{0}^{\tau}\left\|u\left(\tau^{\prime}\right)\right\|_{B_{p, 1}^{2+\frac{1}{p}}} d \tau^{\prime}\right) d t \geq 1\right\} .
$$

Applying inequality (4.5) yields that for $t<\min \left\{T^{\star}, T^{\star \star}\right\}$,

$$
\|w(t)\|_{B_{p, 1}^{1+\frac{1}{p}}} \leq \frac{\left\|w_{0}\right\|_{B_{p, 1}^{1+\frac{1}{p}}} \exp \left(C \int_{0}^{t}\|u(\tau)\|_{B_{p, 1}^{2+\frac{1}{p}}} d \tau\right)}{1-C\left\|w_{0}\right\|_{B_{p, 1}^{1+\frac{1}{p}}} \int_{0}^{t} \exp \left(C \int_{0}^{\tau}\left\|u\left(\tau^{\prime}\right)\right\|_{B_{p, 1}^{2+\frac{1}{p}}} d \tau^{\prime}\right) d t} .
$$

Assumption (4.4) yields that $T<T^{\star \star}$. Now let us show that $T_{v_{0}}^{\star}>T$, arguing by contradiction. In effect, if $T_{v_{0}}^{\star} \leq T$, then according to (4.6), we have for every $t<T_{v_{0}}^{\star}$,

$$
\|w(t)\|_{B_{p, 1}^{1+\frac{1}{p}}} \leq \frac{\left\|w_{0}\right\|_{B_{p, 1}^{1+\frac{1}{p}}} \exp \left(C \int_{0}^{t}\|u(\tau)\|_{B_{p, 1}^{2+\frac{1}{p}}} d \tau\right)}{1-C\left\|w_{0}\right\|_{B_{p, 1}^{1+\frac{1}{p}}} \int_{0}^{t} \exp \left(C \int_{0}^{\tau}\left\|u\left(\tau^{\prime}\right)\right\|_{B_{p, 1}^{2+\frac{1}{p}}} d \tau^{\prime}\right) d t}<+\infty,
$$

which implies that $\|w\|_{B_{p, 1}^{1+\frac{1}{p}}}$ is uniformly bounded on $\left[0, T_{v_{0}}^{\star}\right)$. Since $B_{p, 1}^{1+\frac{1}{p}} \hookrightarrow$ Lip, Theorem 4.2 shows that $v$ may be extended beyond the lifespan time $T_{v_{0}}^{\star}$. The contradiction concludes the proof of Theorem 4.5 .

5. Nonexistence of smooth solitary waves. It is shown in 28 that all these equations in the peakon $b$-family have not only the peakon solutions (1.4) but also multipeakon solutions

$$
u(x, t)=\sum_{k=1}^{N} p_{k}(t) e^{-\left|x-q_{k}\right|},
$$


where the canonical positions $q_{j}$ and momenta $p_{j}$ (with $j=1, \ldots, N$ ) satisfy the following system of ordinary differential equations with discontinuous right-hand side:

$$
\left\{\begin{array}{l}
p_{j}^{\prime}=(b-1) \sum_{k=1}^{N} p_{j} p_{k} \operatorname{sgn}\left(q_{j}-q_{k}\right) e^{-\left|q_{j}-q_{k}\right|} \\
q_{j}^{\prime}=\sum_{k=1}^{N} p_{k} e^{-\left|q_{j}-q_{k}\right|} .
\end{array}\right.
$$

Let $N=2, P=p_{1}+p_{2}, Q=q_{1}+q_{2}, p=p_{1}-p_{2}$ and $q=q_{1}-q_{2}$. Then (5.1) becomes

$$
\begin{cases}P^{\prime}=0, & Q^{\prime}=P\left(1+e^{-|q|}\right), \\ p^{\prime}=\frac{b-1}{2}\left(P^{2}-p^{2}\right) \operatorname{sgn}(q) e^{-|q|}, & q^{\prime}=p\left(1-e^{-|q|}\right) .\end{cases}
$$

In this case, we have two conserved quantities,

$$
P=c_{1}+c_{2}, \quad H:=P^{2}+\frac{p^{2}-P^{2}}{2}\left(1-e^{-|q|}\right)^{b-1}=c_{1}^{2}+c_{2}^{2}
$$

It is also observed that the peakon solution of the $b$-family of equations is not a smooth solution. Actually, one can establish the following result for any travelling wave solutions of the $b$-family of equations.

TheOREM 5.1. There is no nontrivial travelling wave solution $u \in C\left([0, \infty) ; H^{3}\right) \cap$ $C^{1}\left([0, \infty) ; H^{2}\right)$ for (1.2).

Proof. Arguing by contradiction, assume that $w \in H^{3}$ and $u(t, x)=w(x-c t), c \neq 0$ is a strong solution of (1.2). Then we have

$$
c w^{\prime}-c w^{\prime \prime \prime}-(b+1) w w^{\prime}+b w^{\prime} w^{\prime \prime}+w w^{\prime \prime \prime}=0 \quad \text { in } L^{2} .
$$

We find that

$$
\left(c w-c w^{\prime \prime}-\frac{b+1}{2} w^{2}+\frac{b-1}{2}\left(w^{\prime}\right)^{2}+w w^{\prime \prime}\right)^{\prime}=0 \quad \text { in } L_{2}
$$

and therefore

$$
c w-c w^{\prime \prime}-\frac{b+1}{2} w^{2}+\frac{b-1}{2}\left(w^{\prime}\right)^{2}+w w^{\prime \prime}=0 \quad \text { in } H^{1}
$$

or, what is same,

$$
(c-w)\left(w-w^{\prime \prime}\right)-\frac{b-1}{2}\left(w^{2}-\left(w^{\prime}\right)^{2}\right)=0 \quad \text { in } H^{1}
$$

since $w \in H^{3} \subset C_{0}^{2}(\mathbb{R})$. Multiplying this identity with $2 w^{\prime}$ yields that

$$
(c-w)\left(w^{2}-\left(w^{\prime}\right)^{2}\right)^{\prime}-(b-1) w^{\prime}\left(w^{2}-\left(w^{\prime}\right)^{2}\right)=0 .
$$

Since $w \in H^{3} \subset C_{0}^{2}(\mathbb{R})$, we have $w \neq c$, a.e. and $w^{2} \neq\left(w^{\prime}\right)^{2}$, a.e.

Let $w_{0}=w(\xi)=\max _{x \in \mathbb{R}} w(x)>0$. Then taking integration for (5.2) in [ $[\xi, x]$ yields

$$
\int_{\xi}^{x} \frac{d\left(w^{2}-\left(w^{\prime}\right)^{2}\right)}{w^{2}-\left(w^{\prime}\right)^{2}}=\int_{\xi}^{x} \frac{(b-1) d w}{c-w}, \quad x \in \mathbb{R} .
$$

This implies that

$$
|w-c|^{b-1}\left|w^{2}-\left(w^{\prime}\right)^{2}\right|=w_{0}^{2}\left|w_{0}-c\right|^{b-1}, \quad x \in \mathbb{R} .
$$

If we take into account $w, w^{\prime} \rightarrow 0$ as $x \rightarrow \infty$, it is then inferred from (5.3) that

$$
w_{0}^{2}\left|w_{0}-c\right|^{b-1}=0
$$


which also implies from (5.3) that

$$
|w-c|^{b-1}\left|w^{2}-\left(w^{\prime}\right)^{2}\right|=0, \quad x \in \mathbb{R} .
$$

This leads to a contradiction since $w \in H^{3}$.

\section{REFERENCES}

[1] R. Beals, D. H. Sattinger and J. Szmigielski, Acoustic scattering and the extended Korteweg-de Vries hierarchy. Adv. Math. 140 (1998), 190-206. MR1658522 (99i:34120)

[2] A. Bressan and A. Constantin, Global conservative solutions of the Camassa-Holm equation. Arch. Rat. Mech. Anal. 183 (2007), 215-239. MR2278406 (2007j:35183)

[3] A. Bressan and A. Constantin, Global dissipative solutions of the Camassa-Holm equation. Anal. Appl. 5 (2007), 1-27. MR 2288533 (2007k:35394)

[4] J. M. Bony, Calcul symbolique et propagation des singularités pour les équations aux dérivées partielles non linéaires. Ann. Sci. École Norm. Sup. 14 (1981), 209-246. MR631751 (84h:35177)

[5] R. Camassa and D. Holm, An integrable shallow water equation with peaked solitons. Phys. Rev. Letters 71 (1993), 1661-1664. MR1234453 (94f:35121)

[6] R. Camassa, D. Holm and J. Hyman, A new integrable shallow water equation. Adv. Appl. Mech. 31 (1994), 1-33.

[7] J. Y. Chemin, Localization in Fourier space and Navier-Stokes system. Phase Space Analysis of Partial Differential Equations, Proceedings 2004, CRM series, Pisa, pages 53-136. MR2144406 (2006d:35181)

[8] J.-Y. Chemin, Perfect Incompressible Fluids. Oxford Univ. Press, New York, 1998. MR1688875 (2000a:76030)

[9] G. M. Coclite and K. H. Karlsen, On the well-posedness of the Degasperis-Procesi equation. J. Funct. Anal. 233 (2006), 60-91. MR2204675 (2007d:35231)

[10] A. Constantin, The trajectories of particles in Stokes waves. Invent. Math. 166 (2006), 523-535. MR $2257390(2007 \mathrm{j}: 35240)$

[11] A. Constantin, Finite propagation speed for the Camassa-Holm equation. J. Math. Phys. 46 (2005), 023506. MR2121730 (2005h:35303)

[12] A. Constantin, On the scattering problem for the Camassa-Holm equation. Proc. Roy. Soc. London A 457 (2001), 953-970. MR1875310 (2002k:37132)

[13] A. Constantin, Existence of permanent and breaking waves for a shallow water equation: a geometric approach. Ann. Inst. Fourier 50 (2000), 321-362. MR.1775353 (2002d:37125)

[14] A. Constantin and J. Escher, Wave breaking for nonlinear nonlocal shallow water equations. Acta Mathematica 181 (1998), 229-243. MR1668586 (2000b:35206)

[15] A. Constantin and J. Escher, Particle trajectories in solitary water waves. Bull. Amer. Math. Soc. 44 (2007), 423-431. MR2318158 (2008m:76019)

[16] A. Constantin and J. Escher, On the blow-up rate and the blow-up set of breaking waves for a shallow water equation. Math. Z. 233 (2000), 75-91. MR1738352 (2001b:35258)

[17] A. Constantin, V. Gerdjikov and R. Ivanov, Inverse scattering transform for the Camassa-Holm equation. Inverse Probl. 22 (2006), 2197-2207. MR.2277537 (2007j:37119)

[18] A. Constantin and R. S. Johnson, Propagation of very long water waves, with vorticity, over variable depth, with applications to tsunamis. Fluid Dynam. Res. 40 (2008), 175-211. MR2369543 (2009b:76009)

[19] A. Constantin, T. Kappeler, B. Kolev, and P. Topalov, On geodesic exponential maps of the Virasoro group. Ann. Global Anal. Geom. 31 (2007), 155-180. MR2326419 (2008d:58005)

[20] A. Constantin and B. Kolev, Geodesic flow on the diffeomorphism group of the circle. Comment. Math. Helv. 78 (2003), 787-804. MR2016696 (2004k:37163)

[21] A. Constantin and D. Lannes, The hydrodynamical relevance of the Camassa-Holm and DegasperisProcesi equations. Arch. Rational Mech. Anal. 192 (2009), 165-186. MR2481064

[22] A. Constantin and H. P. McKean, A shallow water equation on the circle. Comm. Pure Appl. Math. 52 (1999), 949-982. MR.1686969 (2000m:37146)

[23] A. Constantin and W. A. Strauss, Stability of peakons. Comm. Pure Appl. Math. 53 (2000), 603610. MR 1737505 (2001b:35252) 
[24] R. Danchin, A few remarks on the Camassa-Holm equation. Differential and Integral Equations 14 (2001), 953-988. MR.1827098 (2002e:35204)

[25] R. Danchin, Fourier Analysis Methods for PDEs. Lecture Notes (2005), November 14.

[26] R. Danchin, A note on well-posedness for Camassa-Holm equation. J. Differential Equations 192 (2003), 429-444. MR1990847 (2004h:35197)

[27] H. R. Dullin, G. A. Gottwald, and D. D. Holm, An integrable shallow water equation with linear and nonlinear dispersion. Phys. Rev. Letters 87 (2001), 4501-4504.

[28] A. Degasperis, D. D. Holm, and A. N. W. Hone, A new integral equation with peakon solutions. Theoretical and Mathematical Physics 133 (2002), 1463-1474. MR2001531 (2004d:37098)

[29] A. Degasperis and M. Procesi, Asymptotic integrability, in Symmetry and Perturbation Theory. edited by A. Degasperis and G. Gaeta, World Scientific (1999), 23-37. MR.1844104 (2002f:37112)

[30] J. Escher and B. Kolev, The Degasperis-Procesi equation as a non-metric Euler equation. arXiv:0908.0508.

[31] J. Escher, Y. Liu, and Z. Yin, Global weak solutions and blow-up structure for the DegasperisProcesi equation. J. Funct. Anal. 241 (2006), 457-485. MR2271927 (2008e:35165)

[32] A. Fokas and B. Fuchssteiner, Symplectic structures, their Bäcklund transformation and hereditary symmetries. Physica D 4 (1981), 47-66. MR636470 (84j:58046)

[33] G. Gui, Y. Liu and L. Tian, Global existence and blow-up phenomena for the peakon $b$ family of equations. Indiana University Mathematics Journal 57 (2008), 1209-1234. MR2429090 (2009h:35365)

[34] D. Henry, Infinite propagation speed for the Degasperis-Procesi equation. J. Math. Anal. Appl. 311 (2005), 755-759. MR2168432 (2006d:35225)

[35] D. Henry, Compactly supported solutions of the Camassa-Holm equation. J. Nonl. Math. Phys. 12 (2005), 342-347. MR2160386 (2006b:35286)

[36] D. D. Holm and M. F. Staley, Wave structure and nonlinear balances in a family of $1+1$ evolutionary PDEs. SIAM J. Appl. Dyn. Syst. (electronic) 2 (2003), 323-380. MR.2031278 (2004k:76046)

[37] R. I. Ivanov, On the integrability of a class of nonlinear dispersive wave equations. J. Nonlinear Math. Phys. 12 (2005), 462-468. MR2171998 (2007c:35168)

[38] R. S. Johnson, Camassa-Holm, Korteweg-de Vries and related models for water waves. J. Fluid Mech. 455 (2002), 63-82. MR1894796 (2003b:76026)

[39] B. Kolev, Poisson brackets in hydrodynamics. Discrete Contin. Dyn. Syst. 19 (2007), 555-574. MR 2335765 (2008j:37142)

[40] B. Kolev, Lie groups and mechanics: an introduction. J. Nonlinear Math. Phys. 11 (2004), 480-498. MR2097659 (2005f:70024)

[41] M. Lakshmanan, Integrable nonlinear wave equations and possible connections to tsunami dynamics. Tsunami and nonlinear waves, Springer, Berlin, 49 (2007), 31-49. MR.2364923 (2008m:35309)

[42] J. Lenells, Traveling wave solutions of the Degasperis-Procesi equation. J. Math. Anal. Appl. 306 (2005), 72-82. MR2132889 (2006a:35271)

[43] Z. Lin and Y. Liu, Stabiltiy of peakons for the Degasperis-Procesi equation. Comm. Pure Appl. Math. LXII (2009), 125-146. MR2460268

[44] Y. Liu and Z. Yin, Global existence and blow-up phenomena for the Degasperis-Procesi equation. Comm. Math. Phys. 267 (2006), 801-820. MR2249792 (2007f:35251)

[45] Y. Liu and Z. Yin, On the blow-up phenomena for the Degasperis-Procesi equation. Int. Math. Res. Not., (2007), Art. ID rnm117, 22pp. MR2380010 (2009g:35277)

[46] H. Lundmark, Formation and dynamics of shock waves in the Degasperis-Procesi equation. J. Nonlinear Sci. 17 (2007), 169-198. MR2314847(2008f:35353)

[47] H. Lundmark and J. Szmigielski, Multi-peakon solutions of the Degasperis-Procesi equation. Inverse Problems 19 (2003), 1241-1245. MR2036528(2004m:35279)

[48] Y. Matsuno, Multisoliton solutions of the Degasperis-Procesi equation and their peakon limit. Inverse Problems 21 (2005), 1553-1570. MR2173410 (2006g:35241)

[49] O. Mustafa, A note on the Degasperis-Procesi equation. J. Nonl. Math. Phys. 12 (2005), 10-14. MR:2122861 (2005h:35310)

[50] G. Rodriguez-Blanco, On the Cauchy problem for the Camassa-Holm equation. Nonlinear Anal. 46 (2001), 309-327. MR.1851854 (2002i:35172)

[51] L. Tian, G. Gui and Y. Liu, On the well-posedness problem and the scattering problem for the DullinGottwald-Holm equation. Comm. Math. Phys. 257 (2005), 667-701. MR2164948 (2006e:35240) 
[52] J. F. Toland, Stokes waves. Topol. Methods Nonlinear Anal. 7 (1996), 1-48. MR1422004 $(97 \mathrm{j}: 35130)$

[53] H. Triebel, Theory of Function Spaces. Monographs in Mathematics. Vol. 78, Birkhäuser Verlag, Basel, 1983. MR781540 (86j:46026)

[54] Z. Xin and P. Zhang, On the weak solutions to a shallow water equation. Comm. Pure Appl. Math. 53 (2000), 1411-1433. MR.1773414 (2001m:35278)

[55] Z. Yin, On the Cauchy problem for an integrable equation with peakon solutions. Illinois J. Math. 47 (2003), 649-666. MR2007229 (2004g:35217) 